- Case Report

\title{
A Diabetic Elderly Man with Finger Ulcer
}

\author{
Noraini Mohamad "*, Salziyan Badrin², Wan Noor Hasbee Wan Abdullah ${ }^{3}$ \\ 'School of Dental Sciences, Health Campus, Universiti Sains Malaysia, Kelantan, Malaysia \\ ${ }^{2}$ Department of Family Medicine, School of Medical Sciences, Health Campus, Universiti Sains Malaysia, Kelantan, Malaysia \\ ${ }^{3}$ Dermatology Department, Hospital Raja Perempuan Zainab 2, Kelantan, Malaysia
}

Fixed cutaneous sporotrichosis is a differential diagnosis that can be considered in diabetic patients who present with a poorly healing ulcer. Although its prevalence is low, it can occur in patients with immunocompromised status. Here we report a case of a 70-year-old man with diabetes mellitus who presented with a 1-month history of an unhealed ulcer over the tip of his left middle finger. He experienced a cat bite over his left middle finger 1 month prior to the appearance of the lesion. A skin biopsy revealed the presence of Sporothrix schenckii. Oral itraconazole $200 \mathrm{mg}$ twice daily was started empirically and the patient showed marked improvement in the skin lesion after 2 months of therapy.

Keywords: Sporotrichosis; Ulcer; Itraconazole; Diabetes Mellitus 


\section{INTRODUCTION}

Sporotrichosis is a chronic granulomatous mycotic infection caused by the dimorphic fungus Sporothrix schenckii. ${ }^{1)}$ Sporotrichosis is an endemic worldwide disease that is prevalent in tropical or subtropical areas and temperate zones with warm and humid climates that favor the growth of saprophytic fungi. ${ }^{1,2)}$ Its worldwide prevalence is unknown since sporotrichosis is not a mandatory reported disease. Nonetheless, it has been reported in the United States, South America (Brazil, Columbia, Guatemala, Mexico, Peru), Asia (China, India, Japan), and Australia. ${ }^{1-3)}$ Approximately one case occurs per 1,000 people in Peru, while 200-250 cases in the United States (one to two per million people) occur annually. ${ }^{1)}$ No sex, age, or race is immune to this infection since its occurrence depends on the fungus in the environment and the mode of entry. The higher prevalence among males in most reported cases is attributed to their higher risk of exposure due to the nature of their occupations rather than sex-specific susceptibility. Traumatic injury, which most often occurs during outdoor activities such as farming, gardening, and animal husbandry, is the most common route for inoculation of $S$. schenckii. ${ }^{2)}$ Exposed body parts, the extremities in particular, are the most frequently involved; the upper limbs are more commonly affected than the lower limbs. ${ }^{1,3-5)}$

The mode of transmission of sporotrichosis is through the traumatic implantation of $S$. schenckii from contaminated thorns, hay stalks, barbs, soil, and splinters as well as bizarre roadside injuries leading to cutaneous infection. ${ }^{1)}$ Human infections are also associated with insect stings, fish handling, and the bites of cats, birds, dogs, horses, reptiles, and rats, although clinical symptoms may be lacking in these animals. $^{1,6)}$

\section{CASE REPORT}

A 70-year-old man with a 20-year history of diabetes mellitus presented with a 1-month history of a painful ulcerative lesion over the tip of his left middle finger. The lesion started as a small blister at the distal part of his left middle finger that eventually ruptured and became ulcerated. There was serous discharge that did not smell foul. The patient had experienced a cat bite over his finger 1 month prior to the lesion's appearance. He sought treatment at private clinics twice and was given multiple courses of broad-spectrum antibiotics including oral augmentin, but no improvement was seen. He underwent wound dressing at local health clinics daily and treatment for a diabetic ulcer. However, the lesion persisted by becoming enlarged and reaching the interphalangeal joint area. The patient felt pain in that area.

On examination, his vital signs were within the normal range and his capillary blood glucose was $7.9 \mathrm{mmol} / \mathrm{L}$. Examination of his left middle finger showed an ulcerated lesion that was tender on palpation, irregular in margin, measured approximately $2 \times 2 \mathrm{~cm}$ with serous and bloody discharge. The skin surrounding the lesion looked normal with no signs of inflammation (Figure 1A, B). There were no palpable nodules over the dorsum of the left hand or the left forearm and no palpable lymph nodes.

A full blood panel revealed a hemoglobin level of $13.0 \mathrm{~g} / \mathrm{dL}$, white blood count of $6.0 \times 10^{9} / \mathrm{L}$ and platelet count of $165 \times 10^{9} / \mathrm{L}$. His baseline liver function and renal function test results were in the normal range. His latest hemoglobin Alc result was 7.9\%. A skin biopsy taken from the lesion showed irregular epidermal hyperplasia with hyperkeratosis and parakeratosis. Inflammatory cell infiltration was seen within the epidermis. The dermal layers showed marked acute and chronic inflammatory cell infiltration with histiocytes. The culture media showed growth of $S$. schenckii. Culture and sensitivity tests for Mycobacterium tuberculosis were negative.

Oral itraconazole $200 \mathrm{mg}$ twice daily was prescribed for 4 months; after 2 months, the lesion showed marked improvement. The ulcer completely disappeared after 3 months of treatment (Figure 2), while the treatment was continued for 1 more month.

Informed consent was obtained from the patient for publication of this case report and the accompanying images.
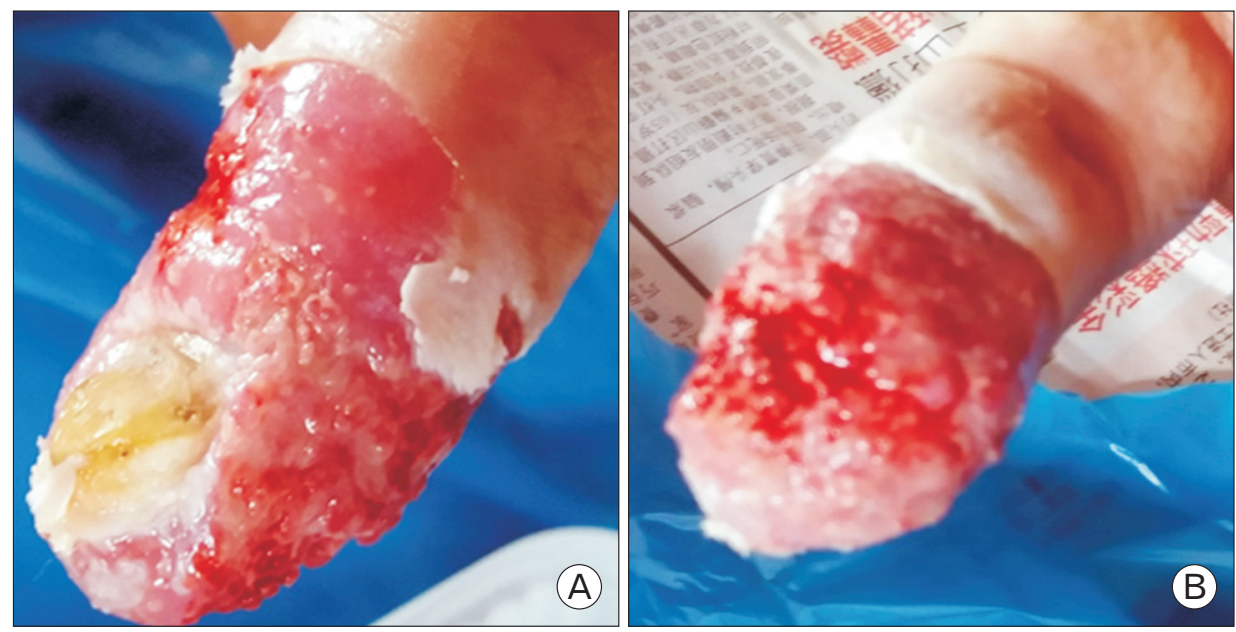

Figure 1. (A, B) Ulcerative lesion over the distal part of the left middle finger. 


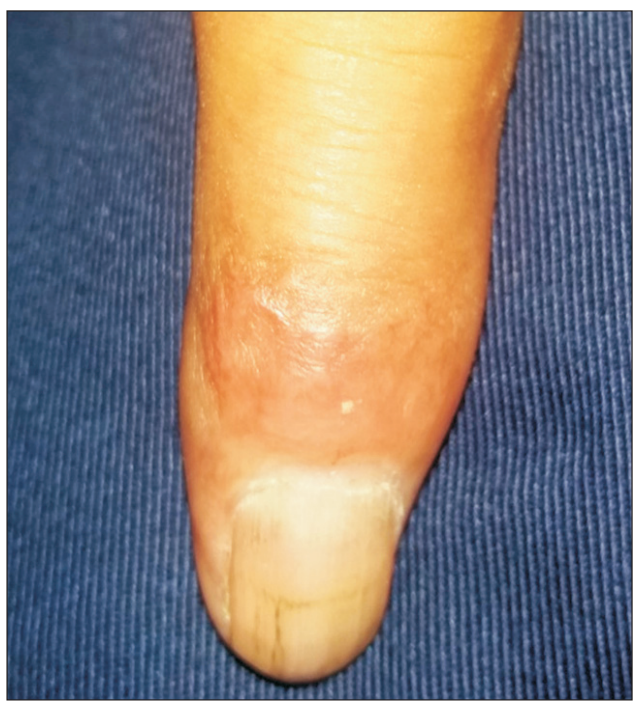

Figure 2. Healed ulcer with scarring over the distal part of the left middle finger after 3 months of oral itraconazole.

\section{DISCUSSION}

Sporotrichosis infections can be cutaneous or extracutaneous. Cutaneous infections are most common and are subclassified into lymphocutaneous and fixed cutaneous types. ${ }^{7)}$ Lymphocutaneous sporotrichosis is the most common variety, accounting for $70 \%-80 \%$ of cases of cutaneous sporotrichosis. ${ }^{1)}$ Lymphocutaneous disease results from a lymphangitic spread of an infection. ${ }^{7)}$ Fixed cutaneous sporotrichosis occurs less commonly and is characterized by localized lesions at the inoculation site. ${ }^{1)}$ Extracutaneous or systemic sporotrichosis occurs from a hematogenous spread from the primary inoculation site, lymph node, or more commonly pulmonary disease in immunosuppressed patients. ${ }^{1)}$ Dissemination to other organs and tissues such as the bones and joints appears to occur more often in patients with an immunosuppressive condition such as alcoholism, acquired immune deficiency syndrome , diabetes, and long-term use of corticosteroids. ${ }^{4)}$

The exact incubation period of the organism remains unknown and may range from a few days to a few months, with the average being 3 weeks. ${ }^{1)}$ After exposure to a variable incubation period, the papulonodule at the inoculation site will be progressively enlarged and may later ulcerate (fixed cutaneous sporotrichosis) or multiple nodules will appear proximally along the lymphatics (lymphocutaneous sporotrichosis). ${ }^{1)}$ In this case, the patient had a history of a cat bite over his left middle finger 1 month prior to the lesion's appearance. There were no palpable lymph nodes along the lymphatic channels over the dorsum of his left hand or left forearm, so the diagnosis of fixed cutaneous sporotrichosis of the left finger was made. After a further probe into his history, he mentioned that the cat had unhealed skin lesions. A study in Rio de Janeiro reported that cats were an important vehicle in the transmission of $S$. schenckii: of the 178 patients diagnosed with sporotrichosis, 156 reported having professional contact with cats with spo- rotrichosis; of those, 97 had a history of a cat scratch or bite, indicating that the feline transmission of sporotrichosis was associated with a large and long-lasting disease outbreak. ${ }^{8)}$

The diagnosis of cutaneous sporotrichosis was based on patient history, clinical examination, and laboratory analysis for the determination of sporotrichosis that included direct examination of tissue biopsy specimens. ${ }^{3,9)}$ The culture of $S$. schenckii in artificial media remains the gold standard for the diagnosis of sporotrichosis. ${ }^{1)}$ However, clinical suspicion is the key to the early diagnosis of cutaneous sporotrichosis. Furthermore, in this case, the diagnosis of fixed cutaneous sporotrichosis required differentiation from cutaneous tuberculosis, squamous cell carcinoma, cutaneous leishmaniasis, and pyoderma gangrenosum. ${ }^{1)}$ Apart from that, this patient had diabetes, so an unhealed diabetic ulcer was mistakenly diagnosed.

The majority of patients with a sporotrichosis infection will require treatment to achieve resolution since its spontaneous recovery is extremely rare. There are various recommended treatment options, most of which are given empirically. Guidelines for the management of patients with sporotrichosis were prepared in 2007 by an expert panel of the Infectious Diseases Society of America. ${ }^{10)}$ These guidelines are primarily based on case reports, retrospective reviews, and nonrandomized trials in which they provide recommendations for the management of lymphocutaneous, cutaneous, pulmonary, osteoarticular, meningeal, and disseminated sporotrichosis. The treatment duration of sporotrichosis is usually prolonged and expensive: 3-6 months is needed for cutaneous sporotrichosis, but any treatment must be continued for 4-6 weeks after clinical remission is achieved to ensure a complete cure. The treatment of choice for uncomplicated cutaneous sporotrichosis infection includes itraconazole $200-400 \mathrm{mg} / \mathrm{d}$, fluconazole $400-800 \mathrm{mg} / \mathrm{d}$, terbinafine $500 \mathrm{mg} / \mathrm{d}$ (250 mg twice daily), and a saturated solution of potassium iodide in increasing doses. ${ }^{1,10)}$

In conclusion, the diagnosis of sporotrichosis can be considered a differential diagnosis in cases of antibiotic-refractory or poorly healing ulcers, especially in patients with diabetes.

\section{CONFLICT OF INTEREST}

No potential conflict of interest relevant to this article was reported.

\section{REFERENCES}

1. Mahajan VK. Sporotrichosis: an overview and therapeutic options. Dermatol Res Pract 2014;2014:272376.

2. Chakrabarti A, Bonifaz A, Gutierrez-Galhardo MC, Mochizuki T, Li S. Global epidemiology of sporotrichosis. Med Mycol 2015;53:3-14.

3. Mahajan VK, Sharma NL, Sharma RC, Gupta ML, Garg G, Kanga AK. Cutaneous sporotrichosis in Himachal Pradesh, India. Mycoses 2005;48:25-31.

4. Da Rosa AC, Scroferneker ML, Vettorato R, Gervini RL, Vettorato G, Weber A. Epidemiology of sporotrichosis: a study of 304 cases in Brazil. J Am Acad Dermatol 2005;52(3 Pt 1):451-9. 
5. Sharma NL, Sharma RC, Gupta ML, Singh P, Gupta N. Sporotriciiosis study of 22 cases from Himachal Pradesh. Indian J Dermatol Venereol Leprol 1990;56:296.

6. Werner AH, Werner BE. Sporotrichosis in man and animal. Int J Dermatol 1994;33:692-700.

7. Miller SD, Vinson RP, Libow LF, Elston DM. Dermatologic manifestations of sporotrichosis [Internet]. New York (NY): Medscape; 2016 [cited 2016 Dec 20]. Available from: http://emedicine.medscape.com/ article/1091159-overview.

8. Barros MB, Schubach Ade O, do Valle AC, Gutierrez Galhardo MC,
Conceicao-Silva F, Schubach TM, et al. Cat-transmitted sporotrichosis epidemic in Rio de Janeiro, Brazil: description of a series of cases. Clin Infect Dis 2004;38:529-35.

9. Barros MB, de Almeida Paes R, Schubach AO. Sporothrix schenckii and sporotrichosis. Clin Microbiol Rev 2011;24:633-54.

10. Kauffman CA, Bustamante B, Chapman SW, Pappas PG; Infectious Diseases Society of America. Clinical practice guidelines for the management of sporotrichosis: 2007 update by the Infectious Diseases Society of America. Clin Infect Dis 2007;45:1255-65. 